\title{
Update on long-term efficacy and safety of dapagliflozin in patients with type 2 diabetes mellitus
}

\author{
Aris Liakos, Thomas Karagiannis, Eleni Bekiari, Panagiota Boura and Apostolos Tsapas
}

\begin{abstract}
Sodium-glucose cotransporter 2 (SGLT2) inhibitors are a novel class of antihyperglycaemic agents with an insulin-independent mode of action. Dapagliflozin is a member of the SGLT2 inhibitors class that has received marketing authorization in Europe and the US for use in patients with type 2 diabetes. This review summarizes current evidence from clinical trials assessing the clinical efficacy and safety of dapagliflozin, and presents data regarding its cost-effectiveness. Treatment with dapagliflozin results in similar reduction in haemoglobin $A_{1 c}$ with other oral antihyperglycaemic drugs, which is preserved over 4 years of treatment. However, compared with most antidiabetic agents, dapagliflozin provides additional clinical benefits including body weight loss and blood pressure reduction. Moreover, treatment with dapagliflozin does not increase risk for hypoglycaemia, but is associated with increased incidence of mild to moderate urinary and genital tract infections. A pivotal outcomes trial of dapagliflozin is expected to clarify its effect on cardiovascular endpoints, whilst a causative relationship between dapagliflozin and select malignancies is unlikely. Finally, based on recent economic evaluations dapagliflozin seems to be a cost-effective option for type 2 diabetes in some settings.
\end{abstract}

Keywords: BMS512148, cost-effectiveness, dapagliflozin, Farxiga ${ }^{\circledR}$, Forxiga $^{\circledR}$, sodium-glucose cotransporter 2 (SGLT2), type 2 diabetes mellitus, Xigduo ${ }^{\circledR}$

\section{Introduction}

Type 2 diabetes mellitus (T2DM) has reached epidemic proportions affecting 56 million people in Europe, accounting for $8.5 \%$ of the adult population. Prevalence of T2DM is expected to increase in the following years with projections suggesting that 69 million Europeans will be affected by 2035 (10.3\% of the adult population) [International Diabetes Federation, 2013]. The pathophysiology of T2DM is characterized by insulin resistance and progressive loss of $\beta$-cell function. Treatment is based on the use of therapeutic agents that address these problems. Nevertheless, available therapeutic options have also significant limitations, such as increased risk of hypoglycaemia with sulphonylureas and insulin. Moreover, management of comorbidities including obesity and hypertension poses unique challenges.

Sodium-glucose cotransporter 2 (SGLT2) inhibitors are a novel class of antihyperglycaemic agents with an insulin-independent mode of action. Glucose filtered in the glomerulus is primarily reabsorbed in the $S 1$ segment of the proximal convoluted tubule through the SGLT2. Pharmacologic inhibition of the SGLT2 results in glucosuria, thus lowering blood glucose levels. Dapagliflozin is a member of the SGLT2 inhibitors class that has received marketing authorization in Europe and the US. Dapagliflozin is indicated along with diet and exercise as monotherapy for patients with T2DM who are intolerant to metformin or for whom metformin is contraindicated. It can also be used as an adjunct to other glucose-lowering agents including insulin for patients with inadequate glycaemic control. In the US, the recommended starting dose for dapagliflozin is $5 \mathrm{mg}$ once daily taken in the morning, which can be increased to $10 \mathrm{mg}$ for patients requiring additional glycemic control [AstraZeneca Pharmaceuticals and Bristol-Myers
Ther Adv Endocrinol

Metab

2015, Vol. 6(2) 61-67

DOI: $10.1177 /$

2042018814560735

(c) The Author(s), 2014. Reprints and permissions: http://www.sagepub.co.uk/ journalsPermissions.nav
Correspondence to: Apostolos Tsapas, MD, PhD, MSc

Associate Professor of Medicine, Clinical Research and Evidence-Based Medicine Unit, Second Medical Department Aristotle University Thessaloniki, Hippokratio General Hospital, 49 Konstantinoupoleos Street, 54642, Thessaloniki, Greece atsapas@auth.gr

Aris Liakos, MD, MSc Thomas Karagiannis, MD MSc

Eleni Bekiari, MD PhD Clinical Research and Evidence-Based Medicine Unit, Second Medical Department, Aristotle University Thessaloniki, Thessaloniki, Greece

Panagiota Boura, MD PhD Second Medical Department, Aristotle University Thessaloniki, Thessaloniki, Greece 
Squibb, 2014]. Nevertheless, the approved summary of product characteristics in Europe suggests that dapagliflozin is initiated at a daily dose of $10 \mathrm{mg}$ [European Medicines Agency, 2014a]. A fixed-dose combination of twice daily dapagliflozin $5 \mathrm{mg}$ with metformin 850 or $1000 \mathrm{mg}$ is also commercially available in Europe [European Medicines Agency, 2014b]. A similar, once daily, extended release formulation of dapagliflozin with metformin was recently approved in the US.

\section{Glycaemic efficacy}

\section{Monotherapy}

Current guidelines advocate the use of metformin as first-line treatment, while other hypoglycaemic drugs are indicated only when metformin is considered inappropriate due to intolerance [Inzucchi et al. 2012; Garber et al. 2013]. When used as monotherapy, treatment with dapagliflozin $10 \mathrm{mg}$ for 12 weeks was equally effective with metformin in reducing haemoglobin $\mathrm{A}_{1 \mathrm{c}}\left(\mathrm{HbA}_{1 \mathrm{c}}\right.$ ) (mean difference $0.12 \%$ [ $95 \%$ confidence interval (CI): -0.41 to 0.17 ]) [List et al. 2009]. Nevertheless, there is a paucity of headto-head trials comparing dapagliflozin with other antidiabetic agents as first-line treatment.

\section{Second-line treatment}

For patients already treated with background metformin use of dapagliflozin, reduced $\mathrm{HbA}_{1 \mathrm{c}}$ compared with glipizide (mean difference $-0.30 \%$; $95 \%$ CI -0.51 to -0.09 ) over 4 years of treatment [Langkilde et al. 2013]. Similarly, as add-on to pioglitazone, dapagliflozin provided sustained glycaemic control compared with placebo over 48 weeks of treatment (difference versus placebo in $\mathrm{HbA}_{1 \mathrm{c}}-0.67 \%$; $95 \%$ CI -0.88 to -0.46) [Rosenstock et al. 2012].

Unfortunately, there are no head-to-head trials directly comparing dapagliflozin with dipeptidyl peptidase-4 (DPP4) inhibitors or glucagon-like peptide-1 (GLP1) analogues as add-on therapy. Nevertheless, some indirect inferences can be drawn based on findings from Bayesian network metaanalyses. As an adjunct to metformin, mean difference in $\mathrm{HbA}_{1 \mathrm{c}}$ for dapagliflozin versus DPP4 inhibitors was $-0.08 \%$ [ $95 \%$ credible interval (CrI) -0.25 to 0.10 ] [Goring et al. 2014], while in patients treated with sulphonylurea monotherapy, addition of dapagliflozin provided similar glycaemic efficacy to GLP1 analogues (mean difference in $\mathrm{HbA}_{1 \mathrm{c}}$ $0.11 \%$; $95 \%$ CrI -0.18 to 0.40 ) [Orme et al. 2014].

\section{Third-line treatment}

When dapagliflozin was used on top of metformin plus sitagliptin, a sustained reduction in $\mathrm{HbA}_{1 \mathrm{c}}$ of $0.6 \%(95 \%$ CI -0.8 to -0.4$)$ was noted compared with placebo after 48 weeks of treatment [Jabbour et al. 2014]. Moreover, in patients treated with basal insulin at a dose of at least 30 international unit (IU)/day and up to two other antidiabetic agents, addition of dapagliflozin 10 mg effectively reduced $\mathrm{HbA}_{1 \mathrm{c}}$ compared with placebo over 2 years of treatment $(-0.35 \% ; 95 \%$ CI -0.55 to -0.15 ) [Wilding et al. 2013].

\section{Body weight reduction}

As monotherapy, dapagliflozin was equally effective with metformin in reducing body weight $(-1.00 \%$; 95\% CI -2.04 to 0.04$)$ [List et al. 2009]. In patients already treated with metformin, use of dapagliflozin was associated with a significant weight loss of $-5.07 \mathrm{~kg}$ (95\% CI -6.21 to -3.93) compared with glipizide [Langkilde et al. 2013]. Similar results were observed for use of dapagliflozin as add-on to pioglitazone for 48 weeks $(-2.30 \mathrm{~kg}$; $95 \% \mathrm{CI}-3.37$ to $-1.23 \mathrm{com}-$ pared with placebo) [Rosenstock et al. 2012]. Of note, based on the results of a network meta-analysis, treatment with dapagliflozin in patients inadequately controlled on sulfonylurea alone resulted in significant body weight reduction compared with placebo $(-1.54 \mathrm{~kg} ; 95 \% \mathrm{CrI}-2.16$ to -0.92$)$, in contrast to GLP1 analogues $(-0.65 \mathrm{~kg} ; 95 \%$ CrI -1.37 to 0.07$)$ and DPP4 inhibitors $(0.57 \mathrm{~kg}$; $95 \%$ CrI 0.09 to 1.06 ) [Orme et al. 2014]. Finally, when used as third-line therapy as adjunct to metformin and sitagliptin, dapagliflozin resulted in significant weight loss compared with placebo $(-2.1 \mathrm{~kg} ; 95 \%$ CI -3.2 to -1.0$)$ [Jabbour et al. 2014].

In a study utilizing dual-energy X-ray absorptiometry, Bolinder and colleagues explored whether weight loss is accounted for by changes in fat or fluid components. Almost two-thirds of the observed body weight reduction with dapagliflozin was attributed to loss of fat mass, which is probably related to the caloric loss induced by glucosuria [Bolinder et al. 2012].

\section{Blood pressure reduction}

A recent meta-analysis investigated the effects of SGLT2 inhibitors on blood pressure [Baker et al. 2014]. Dapagliflozin was associated with a reduction in systolic blood pressure of $3.78 \mathrm{~mm}$ 
Hg (95\% CI -4.49 to -3.07$)$. Similarly, diastolic blood pressure was also reduced by 1.41 $\mathrm{mm} \mathrm{Hg}(95 \% \mathrm{CI}-1.80$ to -0.96$)$. These effects are attributed to osmotic diuresis associated with use of dapagliflozin and were corroborated in 2 trials using 24-hour ambulatory blood pressure monitoring [Iqbal et al. 2014]. Changes in blood pressure were observed as early as 12 weeks after initiation of treatment with dapagliflozin and were similar to those induced by hydrochlorothiazide [Lambers Heerspink et al. 2013].

\section{Hypoglycaemia}

Based on pooled results from 3 studies, dapagliflozin did not increase incidence of hypoglycaemia compared with other antidiabetic agents [odds ratio (OR) $0.49 ; 95 \%$ CI 0.18 to 1.39 ] [Vasilakou et al. 2013]. Incidence of hypoglycaemia was approximately 10 -fold less with dapagliflozin relative to glipizide [Langkilde et al. 2013]. Of note, a lower dose of insulin or an insulin secretagogue may be required to reduce the risk of hypoglycaemia when these are used in combination with dapagliflozin [European Medicines Agency, 2014a].

\section{Urinary and genital tract infections}

Treatment with dapagliflozin $10 \mathrm{mg}$ was associated with an increased incidence of urinary tract infections (UTIs) (OR 1.43; 95\% CI 1.05 to 1.94) based on pooled data from 12 placebo-controlled trials [Vasilakou et al. 2013]. These events were mild to moderate in intensity and were more common in females than males, while pyelonephritis was uncommon and with a similar incidence rate $(0.1 \%)$ as in placebo-treated patients [US Food and Drug Administration, 2011]. Discontinuation rates due to UTIs were generally low $0.3 \%$ for dapagliflozin-treated and $0.1 \%$ for placebo-treated subjects). Events suggestive of UTI were evaluated by means of a urine culture in $39-50 \%$ of patients allocated to dapagliflozin and in $50 \%$ of patients treated with placebo. Approximately twothirds of these urine cultures turned out positive for common pathogens identified in patients with T2DM and UTI [Johnsson et al. 2013].

Moreover, treatment with dapagliflozin was associated with a marked increase of genital tract infections (OR versus placebo 3.48; 95\% CI 2.33 to 5.20) [Vasilakou et al. 2013]. The most commonly reported events were vulvovaginal mycotic infections in females and balanitis in males; however none of them was classified as serious [European Medicines Agency, 2012].

\section{Cardiovascular safety}

A meta-analysis of cardiovascular events submitted by the sponsor to the European Medicines Agency included safety data collected in the dapagliflozin clinical development programme through to 15 July 2011. Based on a total of 145 events, dapagliflozin did not increase the hazard ratio (0.82; $95 \%$ CI 0.58 to 1.15$)$ for the composite cardiovascular endpoint (defined as time to first event of cardiovascular death, myocardial infarction, stroke, or hospitalization for unstable angina) compared with control arms [European Medicines Agency, 2012]. Similar findings were reported in a meta-analysis of 14 trials (OR $0.73 ; 95 \%$ CI 0.46 to 1.16 ) [Vasilakou et al. 2013]. Nevertheless, the long-term effect of dapagliflozin on cardiovascular outcomes will be elucidated after completion of an ongoing placebo-controlled trial that is expected to enroll more than 17,000 patients with T2DM at high risk for cardiovascular events. The primary outcome of this pivotal trial is a composite cardiovascular endpoint including cardiovascular death, myocardial infarction or ischemic stroke. Hospitalization for unstable angina or heart failure, as well as all-cause mortality, will also be investigated. The trial is expected to be completed by 2019 [ClinicalTrials.gov identifier: NCT01730534].

\section{Neoplasms}

A higher incidence of breast cancer was noted in patients treated with dapagliflozin (nine women, $\mathrm{n}=5501$ as opposed to one patient in the control group, $n=3148$ ). However, study day of diagnosis ranged from day 6 to day 334 [European Medicines Agency, 2012]. Thus this relatively short interval between exposure to study drug and diagnosis of breast cancer mitigates against a causative relationship.

Based on the latest data submitted by the drug sponsors to the US Food and Drug Administration, 10 cases $(0.17 \%)$ of bladder cancer were identified in dapagliflozin-treated subjects $(n=6045)$ compared with 1 case $(0.03 \%)$ in comparator groups $(n=3512)$. Diagnosis of bladder cancer was mainly driven by haematuria. Moreover, risk factors that might contribute to the development of bladder cancer were balanced between 
treatment arms [US Food and Drug Administration, 2013]. Although a causal relationship cannot be definitively excluded, this imbalance may well be attributed to detection bias due to frequent urinalyses in dapagliflozintreated patients experiencing urinary tract infections. As a precautionary measure, co-administration of dapagliflozin with pioglitazone is not currently recommended in Europe [European Medicines Agency, 2014a].

\section{Dapagliflozin in special populations}

\section{Renal impairment}

Dapagliflozin is not recommended for use in patients with moderate to severe renal impairment [estimated glomerular filtration rate (eGFR) $\left.<60 \mathrm{ml} / \mathrm{min} / 1.73 \mathrm{~m}^{2}\right]$. A dedicated randomized, placebo-controlled trial of dapagliflozin in patients with chronic kidney disease (mainly stage 3 , with an eGFR $\geqslant 30$ and $<60 \mathrm{ml} / \mathrm{min} / 1.73 \mathrm{~m}^{2}$ ) showed lack of glycaemic efficacy (placebo-corrected difference in $\mathrm{HbA}_{1 \mathrm{c}}-0.11 \%, p=0.435$ for dapagliflozin $10 \mathrm{mg}$ ) and an imbalance in incidence of bone fractures among the two groups (13 patients treated with dapagliflozin, $\mathrm{n}=168$ versus zero events in the placebo group, $\mathrm{n}=84$ ) after two years of treatment [Kohan et al. 2014].

\section{Elderly patients}

Glycemic efficacy of dapagliflozin in elderly subjects has been explored in a pooled analysis of 12 phase IIb/III studies. The effect of dapagliflozin on $\mathrm{HbA}_{1 \mathrm{c}}$ was slightly attenuated in patients $\geqslant 65$ years of age compared with those $<65$ years-old. After controlling for eGFR, no conclusive evidence was found that age affects efficacy as an independent factor $(p=0.29)$ [Fioretto et al. 2013]. Nevertheless, hypoglycaemia, volume depletion and renal adverse events occurred more often in subjects aged $\geqslant 65$ years.

\section{Patients at risk for volume depletion}

Adverse events related to volume depletion (most commonly hypotension) were observed in $0.8 \%$ of patients treated with dapagliflozin $10 \mathrm{mg}$ as opposed to $0.4 \%$ in placebo-treated subjects. Hypotension due to dapagliflozin-induced osmotic diuresis was more common amongst elderly subjects, patients with moderate renal impairment or subjects treated with loop diuretics [European Medicines Agency, 2012]. Discontinuation of treatment with dapagliflozin is recommended for patients who develop volume depletion.

\section{Are the benefits worth the costs?}

T2DM is a chronic and costly disease with an increasing prevalence in both developed and developing countries. Therapeutic interventions that delay or prevent the development of its longterm complications could confer substantial cost savings to health systems worldwide, which are forced to allocate limited healthcare resources efficiently and evenly among the population [Oliver et al. 2004]. As a consequence, many countries have adopted the implementation of Health Technology Assessments (HTAs) and economic evaluations in the decision-making process for reimbursement of new health technologies [Oliver et al. 2004; Brennan et al. 2006].

In the UK, an HTA assessing the cost-effectiveness of dapagliflozin was commissioned by the National Institute for Health and Care Excellence (NICE) [National Institute for Health and Care Excellence, 2013]. It was based on a simulation economic model submitted by the manufacturer that evaluated the cost-effectiveness of dapagliflozin as dual therapy in combination with metformin, as add-on to insulin, or as triple therapy. For the add-on to metformin analysis, the comparators considered were sulphonylureas, DPP4 inhibitors and pioglitazone. Baseline patient characteristics, clinical effectiveness and adverse event data were taken from relevant randomized controlled trials and a network meta-analysis conducted by the manufacturer. The results of the originally submitted analysis suggested that, as add-on to metformin, dapagliflozin dominated DPP4 inhibitors and pioglitazone both in terms of costs and quality-adjusted Life Years (QALYs) gained, while compared with sulphonylureas it was associated with an incremental cost-effectiveness ratio (ICER) of $£ 2671$ per QALY gained. Nevertheless, the NICE Evidence Review Group and the Decision Support Unit identified several flaws in the original analysis. Thus a revised economic model was submitted and additional changes were applied, resulting in ICER per QALY values of $£ 12,405$ and $£ 13,338$ for the comparisons of dapagliflozin versus sulphonylureas and pioglitazone, respectively. Interestingly, DPP4 inhibitors were associated with higher costs and QALYs than dapagliflozin, but these differences were small ( $£ 16847$ per QALY gained). Regarding add-on treatment to insulin, the NICE 
HTA reported that the ICER for dapagliflozin compared with DPP4 inhibitors was below $£ 20,000$ per QALY in the base case and all sensitivity analyses. Overall, the HTA concluded that dapagliflozin is recommended as add-on to metformin, or in combination with insulin with or without other antidiabetic drugs [National Institute for Health and Care Excellence, 2013].

Additional cost-effectiveness analyses have been recently published for Nordic countries [Sabale et al. 2014] and the Netherlands [van Haalen et al. 2014]. A simulation economic model applying the United Kingdom Prospective Diabetes Study 68 equations [Clarke et al. 2004] to predict the incidence of seven diabetes-related long-term complications was utilized to estimate the costeffectiveness of dapagliflozin compared with sulphonylurea as add-on to metformin in four Nordic countries [Sabale et al. 2014]. The ICER per QALY gained with dapagliflozin was $€ 7944$, $€ 5424, € 4769$ and $€ 6093$ in Denmark, Finland, Norway and Sweden, respectively, suggesting that in these countries dapagliflozin seems a costeffective alternative to sulphonylurea in patients inadequately controlled with metformin alone [Sabale et al. 2014]. In addition, an analysis based on a similar simulation model in the Netherlands suggested that dapagliflozin is a cost-effective treatment option for patients on insulin therapy that have inadequate glycaemic control [van Haalen et al. 2014]. Of note, both analyses were based on short-term data derived from a single trial (a sulphonylurea-controlled [Sabale et al. 2014] and a placebo-controlled trial [van Haalen et al. 2014], respectively), while specific limitations regarding estimation of costs [Sabale et al. 2014] can further reduce their applicability. Moreover, it is questionable whether these results are generalizable to other countries due to country-specific variations in clinical practice and differences in costs of drugs and diabetes-related events. More specifically, an assessment estimating the benefit of dapagliflozin conducted by the Institute for Quality and Efficiency in Health Care in Germany concluded that it does not confer any additional benefit neither as monotherapy nor as add-on treatment, mainly due to lack of clinically relevant trials comparing dapagliflozin with appropriate comparator therapy [Institute for Quality and Efficiency in Health Care, 2013].

In Australia, the Pharmaceutical Benefits Advisory Committee initially rejected the manufacturer's submission for reimbursement of dapagliflozin on the basis of uncertain comparative clinical effectiveness [Pharmaceutical Benefits Advisory Committee, 2012a, 2012b]. Nevertheless, a resubmission including data from more clinical trials and a cost minimization analysis comparing dapagliflozin with sitagliptin resulted in approval of dapagliflozin for listing for the treatment of patients with T2DM as dual combination therapy with metformin or a sulphonylurea [Pharmaceutical Benefits Advisory Committee, 2013]. Finally, recent economic analyses have been presented at the International Society for Pharmacoeconomics and Outcomes Research 19th Annual International Meeting. Based on these analyses, in the US, dapagliflozin is dominated both in terms of costs and QALYs by the newer SGLT2 inhibitor canagliflozin [Neslusan et al. 2014], whilst it appears to be a cost effective option compared to sulphonylurea as add-on to metformin in Argentina, Chile and Colombia [Elgart et al. 2014a, 2014b].

\section{Conclusion}

In conclusion, dapagliflozin is a new addition in the antidiabetic armamentarium with similar glycaemic efficacy to other oral antihyperglycaemic agents and low risk for hypoglycaemia. Dapagliflozin offers additional clinical benefits including body weight loss and blood pressure reduction. The main safety concern is related to an increased incidence of urinary and genital tract infections. A pivotal outcomes trial of dapagliflozin is expected to clarify its effect on cardiovascular endpoints.

\section{Funding}

This research received no specific grant from any funding agency in the public, commercial, or notfor-profit sectors.

\section{Conflict of interest statement}

A.T. has received lecture fees from AstraZeneca and Boehringer Ingelheim, and research support from Boehringer Ingelheim, Novartis, Novo Nordisk and Sanofi. The remaining authors declare no conflicts of interest in preparing this article.

\section{References}

AstraZeneca Pharmaceuticals and Bristol-Myers Squibb (2014) Highlights of prescribing information. Farxiga (dapagliflozin) tablets, for oral use. Princeton, NJ: Bristol-Myers Squibb Company. Wilmington, DE: AstraZeneca Pharmaceuticals LP. 
Baker, W., Smyth, L., Riche, D., Bourret, E., Chamberlin, K. and White, W. (2014) Effects of sodium-glucose co-transporter 2 inhibitors on blood pressure: a systematic review and meta-analysis. $\mathcal{F ~} \mathrm{Am}$ Soc Hypertens 8: 262-275.

Bolinder, J., Ljunggren, O., Kullberg, J., Johansson, L., Wilding, J., Langkilde, A. et al. (2012) Effects of dapagliflozin on body weight, total fat mass, and regional adipose tissue distribution in patients with type 2 diabetes mellitus with inadequate glycemic control on metformin. F Clin Endocrinol Metab 97: 1020-1031.

Brennan, A., Chick, S. and Davies, R. (2006) A taxonomy of model structures for economic evaluation of health technologies. Health Econ 15: 1295-1310.

Clarke, P., Gray, A., Briggs, A., Farmer, A., Fenn, P., Stevens, R. et al. (2004) A model to estimate the lifetime health outcomes of patients with type 2 diabetes: the United Kingdom Prospective Diabetes Study (UKPDS) Outcomes Model (UKPDS no. 68). Diabetologia 47: 1747-1759.

Elgart, J., Prestes, M., Gonzalez, L., Aschner, P., Garrido Lecca, S., Aiello, E. et al. (2014a) PDB56 - dapagliflozin versus sulfonylurea as an add-on therapy to metformin: a cost-effectiveness analysis in Colombia. Value Health 17: A247.

Elgart, J., Prestes, M., Gonzalez, L., Asteazaran, S., Aiello, E., Garrido Lecca, S. et al. (2014b) PDB63 dapagliflozin: cost-effectiveness as an add-on therapy to metformin in the treatment of type 2 diabetes (T2DM) in Argentina and Chile. Value Health 17: A248.

European Medicines Agency (2012) Assessment report: Forxiga (Dapagliflozin). Procedure no. EMEA/ H/C/002322. London: European Medicines Agency.

European Medicines Agency (2014a) Forxiga ${ }^{\circledR}$

Summary of product characteristics. London: European Medicines Agency.

European Medicines Agency (2014b) Xigduo ${ }^{\circledR}$ Summary of product characteristics. London: European Medicines Agency.

Fioretto, P., Bruin, T., Johnsson, E., Ptaszynska, A., Parikh, S. and List, J. (2013) Safety and efficacy of the SGLT2 inhibitor dapagliflozin in older patients with type 2 diabetes. Diabetologia 56: S383.

Garber, A., Abrahamson, M., Barzilay, J., Blonde, L., Bloomgarden, Z., Bush, M. et al. (2013) AACE comprehensive diabetes management algorithm 2013. Endocr Pract 19: 327-336.

Goring, S., Hawkins, N., Wygant, G., Roudaut, M., Townsend, R., Wood, I. et al. (2014) Dapagliflozin compared with other oral anti-diabetes treatments when added to metformin monotherapy: a systematic review and network meta-analysis. Diabetes Obes Metab 16: 433-442.

Institute for Quality and Efficiency in Health Care (2013) Dapagliflozin - benefit assessment according to S 35 a Social Code Book V. Cologne, Germany: Institute for Quality and Efficiency in Health Care.

International Diabetes Federation (2013) IDF Diabetes Atlas, 6th edn. Brussels: International Diabetes Federation.

Inzucchi, S., Bergenstal, R., Buse, J., Diamant, M., Ferrannini, E., Nauck, M. et al. (2012) Management of hyperglycemia in type 2 diabetes: a patient-centered approach: position statement of the American Diabetes Association (ADA) and the European Association for the Study of Diabetes (EASD). Diabetes Care 35: 1364-1379.

Iqbal, N., Weber, M., Mansfield, T., List, J. and Ptaszynska, A. (2014) Dapagliflozin lowered ambulatory BP in patients with type 2 diabetes mellitus and hypertension inadequately controlled by a renin-angiotensin system blocker \pm another agent. Diabetologia 57: S334.

Jabbour, S., Hardy, E., Sugg, J. and Parikh, S. and for the Study 10 Group. (2014) Dapagliflozin is effective as add-on therapy to sitagliptin with or without metformin: a 24-week, multicenter, randomized, double-blind, placebo-controlled study. Diabetes Care 37: 740-750.

Johnsson, K., Ptaszynska, A., Schmitz, B., Sugg, J., Parikh, S. and List, J. (2013) Urinary tract infections in patients with diabetes treated with dapagliflozin. F Diabetes Complications 27: 473-478.

Kohan, D., Fioretto, P., Tang, W. and List, J. (2014) Long-term study of patients with type 2 diabetes and moderate renal impairment shows that dapagliflozin reduces weight and blood pressure but does not improve glycemic control. Kidney Int 85: 962-971.

Lambers Heerspink, H., de Zeeuw, D., Wie, L., Leslie, B. and List, J. (2013) Dapagliflozin a glucoseregulating drug with diuretic properties in subjects with type 2 diabetes. Diabetes Obes Metab 15: 853-862.

Langkilde, A., Nauck, M., Prato, S., Duran-Garcia, S., Rohwedder, K., Theuerkauf, A. et al. (2013) Durability of dapagliflozin vs glipizide as add-on therapies in type 2 diabetes inadequately controlled on metformin: 4-year data. Diebetologia 56: S374.

List, J., Woo, V., Morales, E., Tang, W. and Fiedorek, F. (2009) Sodium-glucose cotransport inhibition with dapagliflozin in type 2 diabetes. Diabetes Care 32: 650-657.

National Institute for Health and Care Excellence (2013) Dapagliflozin in combination therapy for treating 
type 2 diabetes. London: National Institute for Health and Care Excellence.

Neslusan, C., Teschemaker, A., Martin, S., Willis, M. and Johansen, P. (2014) PDB62 - Cost-effectiveness analysis of canagliflozin (CANA) versus dapagliflozin (DAPA) as an add-on to metformin (MET) in patients with type 2 diabetes mellitus (T2DM) in the United States. Value Health 17: A248.

Oliver, A., Mossialos, E. and Robinson, R. (2004) Health technology assessment and its influence on health-care priority setting. Int $\mathcal{F}$ Technol Assess Health Care 20: 1-10.

Orme, M., Fenici, P., Lomon, I., Wygant, G., Townsend, R. and Roudaut, M. (2014) A systematic review and mixed-treatment comparison of dapagliflozin with existing anti-diabetes treatments for those with type 2 diabetes mellitus inadequately controlled by sulfonylurea monotherapy. Diabetol Metab Syndr 6: 73.

Pharmaceutical Benefits Advisory Committee (2012a) Public summary document: Dapagliflozin, tablet, $10 \mathrm{mg}$, (as propanediol monohydrate), Forxiga ${ }^{\circledR}$ (dual oral). Canberra: Australian Government, Department of Health, Pharmaceutical Benefits Advisory Committee.

Pharmaceutical Benefits Advisory Committee (2012b) Public summary document: Dapagliflozin, tablet, 10 $m g$, (as propanediol monohydrate), Forxiga ${ }^{\circledR}$ (insulin). Canberra: Australian Government, Department of Health, Pharmaceutical Benefits Advisory Committee.

Pharmaceutical Benefits Advisory Committee (2013) Public summary document: Dapaglilfozin, tablet, 10 $m g$, Forxiga ${ }^{\circledR}$. Canberra: Australian Government, Department of Health, Pharmaceutical Benefits Advisory Committee.

Rosenstock, J., Vico, M., Wei, L., Salsali, A. and List, J. (2012) Effects of dapagliflozin, an SGLT2 inhibitor, on $\mathrm{HbA}_{1 \mathrm{c}}$, body weight, and hypoglycemia risk in patients with type 2 diabetes inadequately controlled on pioglitazone monotherapy. Diabetes Care 35: 1473-1478.

Sabale, U., Ekman, M., Granstrom, O., Bergenheim, K. and McEwan, P. (2014) Costeffectiveness of dapagliflozin (Forxiga ${ }^{\circledR}$ ) added to metformin compared with sulfonylurea added to metformin in type 2 diabetes in the Nordic countries. Prim Care Diabetes. 16 May 2014. DOI: $10.1016 /$ j.pcd.2014.04.007. [Epub ahead of print].

US Food and Drug Administration (2011) FDA briefing document. NDA 202293. Dapagliflozin tablets, 5 and $10 \mathrm{mg}$. Silver Spring, MD: US Food and Drug Administration.

US Food and Drug Administration (2013) FDA briefing document. NDA 202293. Dapagliflozin oral tablets, 5 and $10 \mathrm{mg}$. Silver Spring, MD: US Food and Drug Administration.

Van Haalen, H., Pompen, M., Bergenheim, K., McEwan, P., Townsend, R. and Roudaut, $M$. (2014) Cost effectiveness of adding dapagliflozin to insulin for the treatment of type 2 diabetes mellitus in the Netherlands. Clin Drug Investig 34: 135-146.

Vasilakou, D., Karagiannis, T., Athanasiadou, E., Mainou, M., Liakos, A., Bekiari, E. et al. (2013)

Sodium-glucose cotransporter 2 inhibitors for type 2 diabetes: a systematic review and meta-analysis. Ann Intern Med 159: 262-274.

Wilding, J., Woo, V., Rohwedder, K., Sugg, J. and Parikh, S. and for the Dapagliflozin 006 Study Group (2013) Dapagliflozin in patients with type 2 diabetes receiving high doses of insulin: efficacy and safety over 2 years. Diabetes Obes Metab 16: 124-136.
Visit SAGE journals online http://tae.sagepub.com

(SAGE journals 